For Internal Distribution Only

Accelerator Division

Alternating Gradient Synchrotron Department

BROOKHAVEN NATIONAL LABORATORY

Upton, New York 11973

Accelerator Division

Technical Note

AGS/AD/Tech. Note No. 460

Is the Special Ejection (Cooling Water) System a Major Path by which New Water Activation Enters the Main Magnet Cooling System?

L.A. Ahrens

March 11, 1997 


\title{
Is the Special Ejection (Cooling Water) System a Major Path by which New Water Activation Enters the Main Magnet Cooling System?
}

\author{
L.A. Ahrens
}

\section{Summary}

The Special Ejection cooling water System (SES) as connected during the FY96 high intensity proton run was not the major path for newly activated water from the ring to enter the larger AGS Main Magnet cooling system (MM). This conclusion is based on measurements of the activity in the SES streams coming from and going to the ring and in the equivalent main magnet streams. The measurements are then compared against predictions from a simple model of equilibrium activity in the cooling system. As a consistency check, the decay of the activity for the streams as the HEP program is turned on and turned off (namely going from an idle machine to one extracting $50 \times 10^{12}$ protons every $3.6 \mathrm{sec}$ and vice versa) is also measured and reported.

\section{Introduction}

This note describes aspects of the present configuration of the Special Ejection cooling System and the AGS main magnet cooling system relevant to understanding and estimating certain dose rate measurements. Several such measurements (" $\mathrm{mR} / \mathrm{hr}$ at contact with the pipe") made on the system during the 1996 high intensity proton run are described. A simple model evaluating the expected equilibrium activity levels under particular assumptions is explained. The predictions from that model are compared with the measurements. The point of the exercise is to determine if somehow removing the water exiting the ring via the SES from the rest of the AGS main magnet cooling system would significantly reduce the activation level presently found in the Main Magnet system (and hence reduce the levels created elsewhere by the water in the coolers for that system).

\section{The SES System}

The SES is an auxiliary loop in the much larger AGS main magnet cooling system. The SES tee's off the high pressure side of the main magnet system and ultimately returns into the low pressure side of that system. In between, the water pressure is boosted and the water stream split between the F10 extraction septum and the I10 snake. The SES return includes this water from the F10, and the snake but also takes water which cooled the F5 magnet.(The input for water cooling F5 comes directly from the main magnet system.) The SES return water ends up going into the low pressure side of the main magnet system before the water goes to the coolers. Figure 1 gives a sketch of the systems. Table 1 gives the parameters used in a model for this system to be described. These numbers are accurate only at the 10-20\% level. Their apparent precision reflects only the desire to keep things self consistent. 


\begin{tabular}{||l|l|l|l||}
\hline & $\begin{array}{l}\text { Volume } \\
\text { (gallons) }\end{array}$ & $\begin{array}{l}\text { Incoming } \\
\text { Flows } \\
\text { (gal/min) }\end{array}$ & $\begin{array}{l}\text { Outgoing } \\
\text { Flows } \\
\text { (gal/min) }\end{array}$ \\
\hline \hline Main Magnet System & & & \\
\hline \hline MMtrr "to ring + ring/2" & 1800 & 2634 & 2634 \\
\hline MMfrr "from ring + ring/2" & 1800 & 2634 & 2634 \\
\hline $\begin{array}{l}\text { Cooling Tower (divided arbitrarily into } \\
\text { four chambers) }\end{array}$ & 3200 & $\begin{array}{l}2634(\mathrm{MM}) \\
66(\mathrm{SES})\end{array}$ & $\begin{array}{l}2634(\mathrm{MM}) \\
66(\mathrm{SES})\end{array}$ \\
\hline \hline SES System & Volume & $\begin{array}{l}\text { Incoming } \\
\text { Flows } \\
\text { (gal/min) }\end{array}$ & $\begin{array}{l}\text { Outgoing } \\
\text { Flows } \\
\text { (gal/min) }\end{array}$ \\
\hline \hline SEStr1 (to ring, to S.W. tunnel measur- \\
ing pt) & 40 & 66 & 66 \\
\hline SEStr2 (to outgoing "T") & 22 & 66 & $\begin{array}{l}69(\mathrm{H} 10) \\
27(\mathrm{snake})\end{array}$ \\
\hline SEStr3 (to H10) & 23 & 39 & 39 \\
\hline snake & 200 & 27 & 27 \\
\hline F10+ (to return T with snake) & 24 & 39 & 39 (F10) \\
\hline SESfr1 (from ring 1) & 22 & 66 (snake) & 66 \\
\hline SESfr2 (from ring 2) & 30 & 66 \\
\hline
\end{tabular}

Table 1 - Parameters for the Main Magnet and SES Water System. 


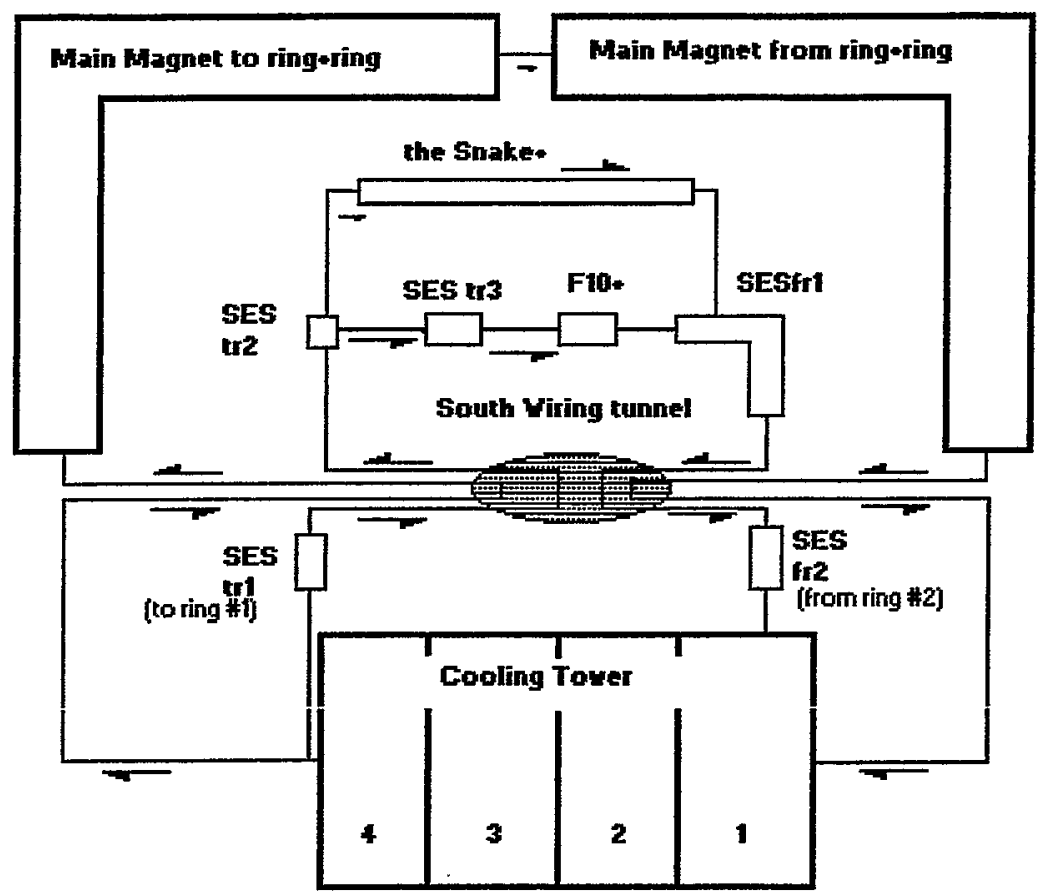

Figure 1 - Sketch of the Main Magnet and SES Cooling Systems.

The relatively low flow in the SES system, combined with the high activity levels in the SES return allow the conjecture that by adding a holding tank in the SES between the ring and the cooling tower, the levels on the larger main magnet system could be substantially reduced. The SES flow is presently 66 gallons/minute (gpm), and if the snake were turned off - the snake is not powered for high intensity running - the flow would be about 40 gpm. To introduce a 20 min delay would require an 800 gallon tank - a tank of modest size.

The SES system configuration is such that radiation from both the SES input to the ring and the return from the ring are easily measured in the "north-east" extension of the South Wiring Tunnel. This region is classified as is a "radiation area"; it is accessible while the beam is "on". Further the geometry is simple - essentially two dimensional and identical for the two lines. A cross section of this tunnel is given in figure 2 . The figure is to scale. It does not show all of the other piping in the tunnel. The water flows in parallel 2.5 inch diameter steel pipes with many tens of feet of straight parallel piping on either side of the measuring point. The only other significant radiation sources in this section of the tunnel are the two (large but somewhat removed) main magnet feed and return water lines. Measuring the levels in these main magnet pipes is also useful, and their longitudinal geometry is similar to that of the SES pipes. 


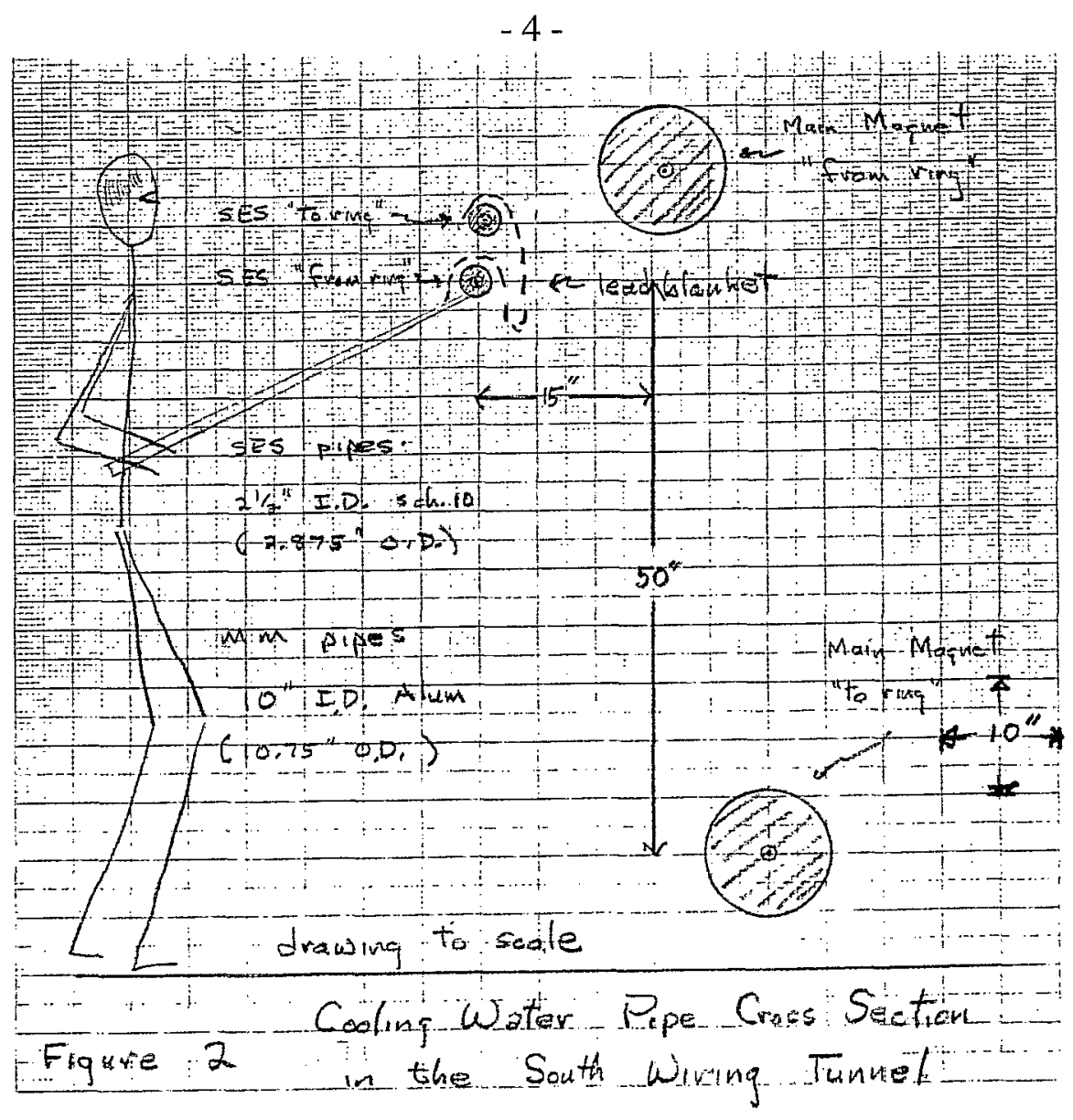

A preliminary survey of the SES lines at this location indicated that with beam "on" the radiation levels were significantly different for the SES "to ring" pipe and the SES "from ring" pipe. Further measurement showed that the radiation level of the water coming from the ring was dominated by immediate beam activity. More precisely, the levels responded to the extracted beam (off-on) and (on-off) with a level change of about $10 \mathrm{mR} / \mathrm{hr}$ (over a few $\mathrm{mR}$ floor) and with a rather precise (about 46 second) time delay between the time the beam state was changed and the time the activity level changes - simply using a "teletector" radiation meter (a long rod with a sensor at one end) for the measurement. Presumably an ion chamber with sufficient gain would see the extracted beam "spill" on radiation from this water although this hasn't been tried. The measured time delay also provides a constraint or check on the flow geometry for a piece of the SES model.

To attempt to prove that the SES is in fact a major player in the Main Ring water activity seen at the cooling tower a prediction of the consequences of this observed activity in SES is necessary. A model to extract an equilibrium estimate for the situation where for example only the cooling of the F10 magnet creates activated water is described next. The point is that at equilibrium the levels in the SES ring output leg translate into levels in the main magnet system, and consequently into levels in the SES ring input leg. The ratio (SES out)/(SES back) from the model prediction and from the real world can then be compared. 


\section{The Model}

The model (which followed discussions with Ed Lessard, and which was constructed in part just to check equivalent predictions he had made - which indeed were born out) replaces the water system by a series of "equivalent" tanks. The volumes of the tanks are set to be equal to the volume of the actual system. The flows between tanks are the actual system flows. The number of tanks in the model is somewhat arbitrary. Main physical systems (which in fact are pipes) are grouped into these tanks. The model assumes each tank is in an equilibrium situation from the point of view of activity - the number of Curies contained is independent of time. Further, the concentration of Curies in any one tank is the same throughout - there is instant mixing. The water flowing out of the tank carries this average activity. The tanks defined for the results below were in part chosen to provide activity predictions at the points where the measurements are actually made in the SES and main ring systems. Figure 1, which gives the "tank" configuration of the model, then has the South Wiring tunnel location as a point between tanks.

A given tank can have several input streams, each with a flow rate (gallons/min) and activity (Curies/gal) which increase the number of Curies inside. The tank can also have several output streams similarly described which reduce the number of Curies in the tank. (These will carry away water with average tank activity). Two other activity-affecting processes are included in the model. If the tank sees the beam, it can gain Curies directly in our initial hypothesis only the F10 tank sees beam. All the tanks lose activity due to decay, the rate being proportional to the number of Curies present in the tank - which is not changing since we are at equilibrium. The rates of creation and decay depend on the unstable ion species being considered (i.e. ${ }^{15} \mathrm{O}$ ).

For each tank an expression stating the equality of the increase in Curies due to the beam to the net decrease due to the flows out of the volume and decays (all proportional to the number of Curies in the volume) less the flows into the volume (proportional to the number of Curies in upstream volumes) can be written down. For the results below a 13 tank model yields 13 equations. $\mathbf{A}=(\mathbf{M}) *(\mathbf{N})$, where $\mathbf{A}$ is the beam activation into each of the 13 volumes (a 13 element vector, [Curies/min]), $\mathbf{N}$ is the number of Curies in each tank (again a 13 element vector [Curies]) and $\mathbf{M}$ is a $13 \times 13$ matrix of flows and decay constants with units [ $\mathrm{min}^{-1}$ ]. Each diagonal element of $\mathbf{M}$ corresponds to one of the volumes in the model and contains the decay constant and the out going flow rates [gallons/min] divided by the tank volume. Off diagonal elements carry the incoming flow rates divided by the upstream tank volume. Each column of the matrix which is the inverse of $\mathbf{M}$ then gives a set of numbers proportional to the number of Curies in each tank for beam activation in one of the tanks. The column associated with F10 activation is evaluated for each of the four most important unstable ions produced. The numbers are scaled by the production rates, and the four columns are added. The relative equilibrium activity in any two tanks is then obtained by dividing the above numbers by the volumes of the respective tanks, and taking the ratios of the results. For the unstable ions and production rates we rely on information taken from A.H. Sullivan's book A Guide to Radiation and Radioactivity Levels near High Energy Particle Accelerators and in particular the table on page 139. 


\section{Results}

With the only water activation source located in the F10 "tank", the model expects at equilibrium that the activity of the SES water going toward the ring would be $3 \%$ of that for SES water coming from the ring. Given this and the short cycle time of the main ring system it is not surprising that the predicted activity in both the "to" and "from" streams of the main magnet water in the South Wiring tunnel are also at the $3 \%$ level. The water from the ring in the main magnet loop is actually predicted to be a bit cooler $(2.7 \%$ of the SES "from ring" water) - reasonable just because the time available for decay is longer. If 10 $\mathrm{mR} / \mathrm{hr}$ were measured at SES "from-the-ring", SES "to-the-ring" should read . $3 \mathrm{mR} / \mathrm{hr}$. For the main magnet pipes predicting an actual reading requires not only predicting the water activity, but also taking account of the fact that the geometry is very different with a 10 inch aluminum pipe rather than a 2.5 inch steel pipe. This geometry factor is determined experimentally (see discussion below) to be about 2.5. For the same water in both types of pipes, the big main magnet pipes give a reading (at contact) which is 2.5 times the reading on an SES pipe. This leads to a prediction in the $10 \mathrm{mR} / \mathrm{hr}$ SES "from ring" (and no other activation sources) situation of $.7 \mathrm{mR} / \mathrm{hr}$ and $.6 \mathrm{mR} / \mathrm{hr}$ for the main magnet "to ring" and "from ring" streams respectively. A just slightly modified version of these numbers (with the assumed rate at SES "from ring" reduced to $9.4 \mathrm{Mr} / \mathrm{hr}$ ) is given in table 2, column 3 .

What is actually measured? In SES "to ring" - $1.5 \mathrm{mR} / \mathrm{hr}$ (five times the F10 only source prediction); in the main magnet "to ring" - $3 \mathrm{mR} / \mathrm{hr}$ (four times predicted); and in main magnet "from ring" - $5 \mathrm{mR} / \mathrm{hr}$ (eight times predicted). These measurements are given in the second column of table 2 . The disagreements with prediction are the basis for the main conclusion of this note.

\begin{tabular}{|l|l|l|l|}
\hline $\begin{array}{l}\text { Measurement Point } \\
\text { in the Model }\end{array}$ & $\begin{array}{l}\text { Measured } \\
\text { Activity, typi- } \\
\text { cal 50Tp ex- } \\
\text { traction }\end{array}$ & $\begin{array}{l}\text { Prediction: As- } \\
\text { sume Activa- } \\
\text { tion @ F10 } \\
\text { only }\end{array}$ & $\begin{array}{l}\text { Prediction: Assume Activa- } \\
\text { tion @ downstream Main } \\
\text { Magnet only }\end{array}$ \\
\hline SES “from ring" & $10 \mathrm{mR} / \mathrm{hr}$ & $\begin{array}{l}9.4 \mathrm{mR} / \mathrm{hr}(\mathrm{de}- \\
\text { fined) }\end{array}$ & $.6 \mathrm{mR} / \mathrm{hr}$ \\
\hline SES “to ring" & $1.5 \mathrm{mR} / \mathrm{hr}$ & $.3 \mathrm{mR} / \mathrm{hr}$ & $1.1 \mathrm{mR} / \mathrm{hr}$ \\
\hline $\begin{array}{l}\text { Main Magnet "to } \\
\text { ring" }\end{array}$ & $3 \mathrm{mR} / \mathrm{hr}$ & $.7 \mathrm{mR} / \mathrm{hr}$ & $2.7 \mathrm{mR} / \mathrm{hr}$ \\
\hline $\begin{array}{l}\text { Main Magnet "from } \\
\text { ring” }\end{array}$ & $5 \mathrm{mR} / \mathrm{hr}$ & $.6 \mathrm{mR} / \mathrm{hr}$ & $4.4 \mathrm{mR} / \mathrm{hr}$ (defined) \\
\hline
\end{tabular}

Table 2 - Measured / Predicted Equilibrium Water Activation. 
The last column in Table 2 gives the predictions from the same model for a situation where the only source of activation to the cooling water is in the "tank" defined for the downstream half of the main magnet system. For columns three and four the cell values labeled as "defined" are adjusted such that the measured levels at the SES "from ring" pipe and the Main Magnet "from ring" are obtained. Then the model predicts the levels to be seen at the SES and Main Magnet "to ring" pipes. Clearly the model has no trouble making acceptable predictions. Further, since the streams returning to the ring reflect the activity at the cooling tower, this interpretation would assign only about $20 \%$ of the activation of the water to the SES "from ring" line.

We next examine to what extent the simple SES "from ring" only source of activity hypothesis is ruled out. Could systematic errors in the measurements invalidate the conclusion? Despite the large fractional disagreement between prediction and measurement, the discrepancies in absolute terms are small. And it is true that the highest dose readings in the tunnel are coming from the SES "from ring" line. Crosstalk from the SES "from ring" pipe could invalidate the conclusion. The inclusion of the predicted and measured activity for the main magnet streams with pipes physically more separated from the SES "from ring" pipe than the other SES pipe is important - perhaps critical. Ignoring any self shielding or shielding from external structures - the "blanket" to be described below or other pipes - a simple $1 / \mathrm{r}$ fall off from the long pipes would result in a reduction in the measured rate due to the SES "from ring" pipe by a factor of 35 [50"/1.44"] at the surface of the Main Magnet "to ring" pipe. The actual reduction with the various shieldings taken into account must be greater than this. Hence the $3 \mathrm{mR} / \mathrm{hr}$ measured at the MM "to ring" pipe was not significantly due to the $(10 \mathrm{mR} / \mathrm{hr})$ SES pipes. A similar $1 / \mathrm{r}$ calculation between the two SES pipes gives only a factor of 3.7, again ignoring all shielding. This would reduce the measured $10 \mathrm{mR}$ at SES "from" only to $2.6 \mathrm{mR}$ at SES "to", which is greater than the measured $1.5 \mathrm{mR}$ there. The rate reduction must fall faster than $1 / \mathrm{r}$. During the measurements lead blanket shielding was draped (see figure 2) to improve isolation between the various pipe measurements. The practical geometry for deploying this resulted in a single layer between the SES pipes, and a double layer between SES "from ring" and the Main Magnet lines. The effectiveness of this blanketing in reducing crosstalk was not determined. The measured radiation levels were typically not stable enough in time to do this. The conclusion being expressed for these measurements then clearly depends on the readings taken on the main magnet pipes.

One auxiliary study is presented. This involves measurements of the decay of the dose rates seen at the surfaces of the four pipes when the beam is inhibited, after having been on steadily for many minutes. All four pipes should yield the same decay rate, which should agree with the prediction for the four expected contributing isotopes. A second piece of information from this data is an experimental determination of the effect of the pipes and enclosed water on the measured dose rates at the pipe surfaces for the two pipe geometries. A single "Ludlum" meter was used for these measurements which were taken by physically cycling through the four pipes, over and over. One cycle took about one minute and the measurements began about 1 minute after the beam was turned off. The one minute initial 
delay is enough time to allow the water in all four pipes to be at the same activity level. The water streams have all come from the cooler and have seen no beam activation after leaving the cooler. The lead blanket shielding described above was in place for this measurement. Figure 3 gives the result from this measurement, where readings were taken for 15 minutes. The early slope for this data (fit to the first three points, extending to 4 minutes from beam off time) gives a decay rate of 3.5 minutes. The time constant for the dominant contributor in the model $\left({ }^{15} \mathrm{O}\right)$ is about 3 minutes.

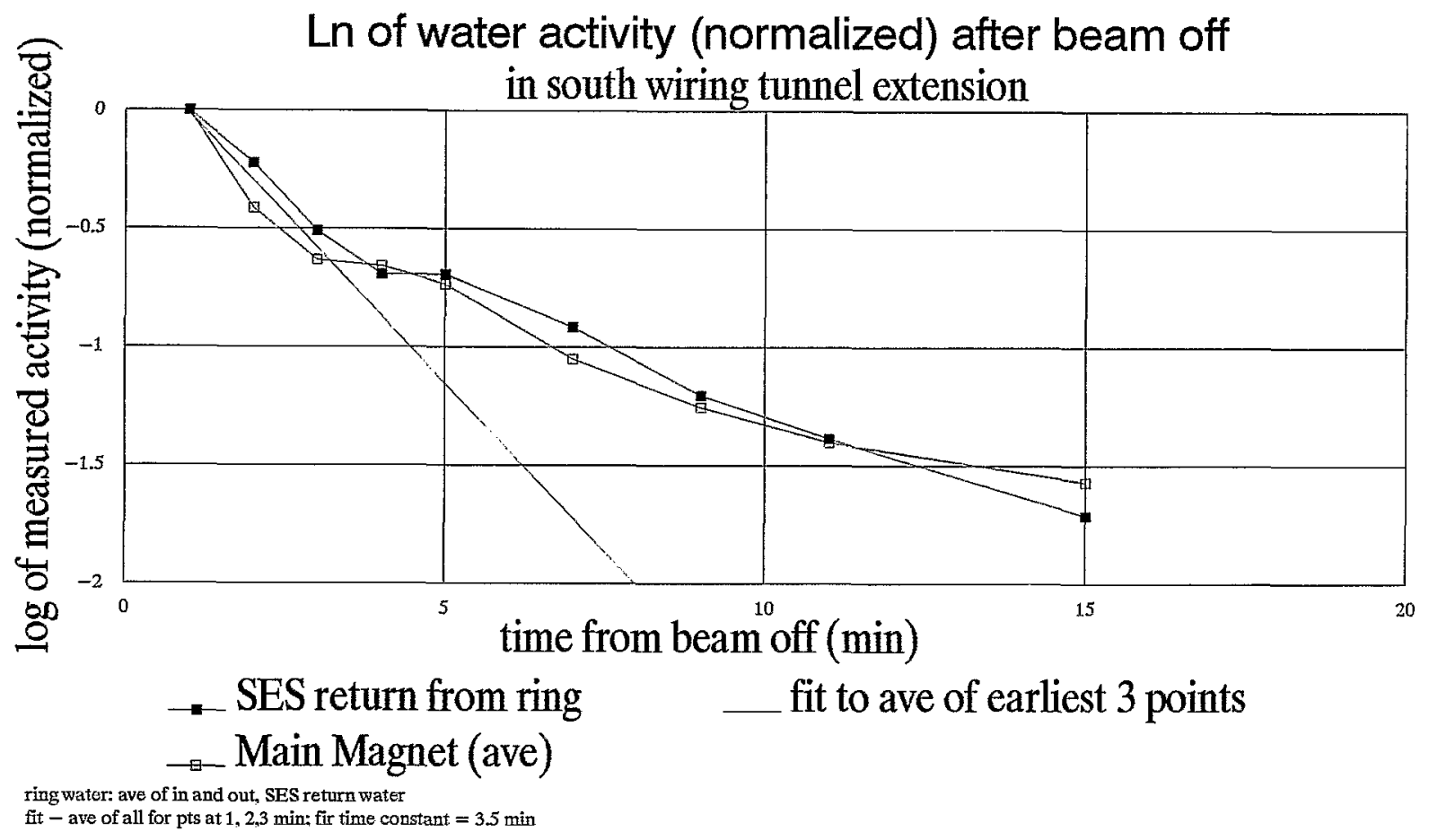

Figure 3 - Decay of the Water Activity in the SES Pipes.

Figure 4 presents two "systematic" properties of the decay rate data set. The upper trace gives the ratio of the measurements at the surface of one of the SES pipes to the measurements at the surface of the Main Magnet pipes. The geometry factor of 2.5 which has been used in the above analysis comes from this data. The careful reader may have notices that only one of the two SES pipes, the "from the ring" pipe has been included so far in this auxiliary study discussion. The reason is that data from the other SES pipe appeared to have an offset from zero. The lower plot in figure 4 resulted from looking more closely at this data. What is shown is the difference between the two SES pipe readings (which one would expect to be zero) divided by the average of readings at the surfaces of the main magnet pipes (which within errors were equal). The SES difference appears to scale with the level at the Main Magnet pipe surface. This might be an indication of the sort of cross talk mentioned above, only in this case presumably from the upper main magnet pipe to the (upper) SES 
pipe. With this explanation the measurement at the Main Magnet pipe is attenuated by a factor of about .15 (from the plot). The $1 / \mathrm{r}$ prediction would be about .3 , so if this is the explanation, in this case the shielding effects introduce an additional factor of 2.

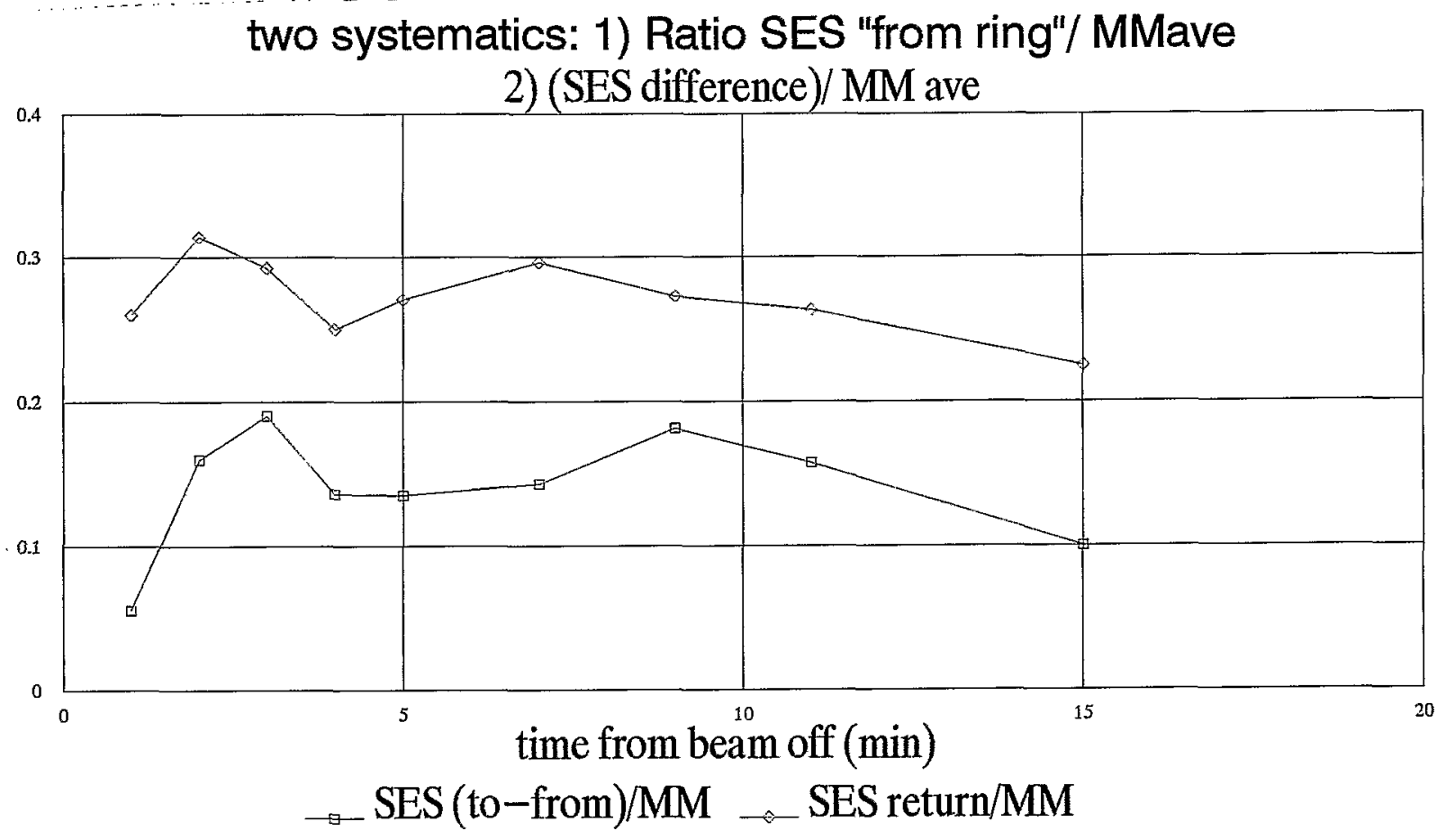

Figure 4 - Systematics in the SES Aux Study.

\section{Further Comments}

This note concludes that the SES as configured for the 1996 high intensity proton run was responsible for $20 \%$ of the activity seen in the main magnet water. Systematic errors in the measurements have been discussed above, and improvements to the measurement technique are certainly possible. Systematic errors in the model have only been explored in one simple way; namely the number of tanks used in the model was increased in the course of the analysis, with no resulting change in the conclusion. The $20 \%$ contribution is not negligible, and a $\mathrm{x} 3$ error (e.g. due to some model systematic) would reverse the conclusion as to the usefulness of adding a holding "cool down" tank.

During the 1997 high intensity run the SES system will be modified to include the water cooling the E20 beam catcher in the return flow. This is the last "trivial" addition to the SES which may contain significant newly activated water - perhaps more this year than last due to more effective use of the catcher. The activity measurements will be made again, and compared with the results from 1996 - namely this note. 
The author appreciates the patience of Ed Dale, and the members of his team in explaining the cooling systems, many times. AGS Operations allowed the measurements to be made, by informing the author of anticipated changes in the machine state both on the long and short time scales. 